\title{
TARDIVE AKATHISIA AND SUICIDE; A CASE REPORT
}

J. Ruiz Navarro ${ }^{1}$, C. García Montero ${ }^{1}$, A. Duque Dominguez, A. Mas Villaseñor ${ }^{1}$, M. Mejía Quiterio ${ }^{1}$, C. Fernández Muñiz', M. Escamilla Espinola ${ }^{2}$.L.. Al Chaal Marcos ${ }^{1}$, F. De La Torre Brasas ${ }^{1}$, N. Echeverría Hernández ${ }^{1}$

${ }^{1}$ Complejo Asitencial de Ávila, Servicio de Psiquiatría, Ávila, Spain

${ }^{2}$ Complejo Asitencial de Ávila, Servicio de Medicina Interna, Ávila, Spain

\section{OBJECTIVES:}

Here we present the case of a patient with tardive drug induced akathisia associated with suicidal behavior. We describe the main characteristics of this movement disorder, the patient's disease progression and his treatment.

\section{BACKGROUND:}

Akathisia is a movement disorder or symptom that is defined as a sense of inner unease and a compelling need to move a body area accompanied by an objective motor component of restlessness. Akathisia straddles the boundary between being a symptom and a sign. Akathisia should be considered a sensorimotor disorder because of the powerful sensory component, which is a defining characteristic of the condition. There are several subtypes of akathisia according to the time of onset in the course of pharmacologic treatment. Tardive akathisia occurs late in the course of pharmacologic treatment and may emerge initially after pharmacologic discontinuation or dosage reduction. This situation has typically been associated with dopamine receptor blockers, such as neuroleptics. The pathophysiology of akathisia is unkown: a blockade of mesocortical dopaminergic pathways and a generalized reduction in dopamine in the brain with compensatory mechanisms (increased noradrenergic activity from the locus ceruleus) are probable hypotheses. Various pharmacological agents has been used for the treatment of akathisia, but evidence on the treatment of tardive akathisia is unsatisfactory. Anxiety, mood disorders and suicidal behavior has been associated with akathisia.

\section{MATERIAL AND METHODS:}

Description of a clinical case and literature review.

\section{RESULTS AND CONCLUSION:}

A 60-year-old male, without previous psychiatric history, with a 2-year history of tardive akathisia induced by clebopride, was admitted to the acute inpatient psychiatry unit of our hospital, because of a suicide attempt (he rushed out the window). His main manifestation was rubbing the hands (predominant upperbody involvement), althought the lower extremities were also involved. He described that feeling as "absolutely unbereable", and it was associated with extreme dysphoria. The score of the Global Clinical Assessment of Akathisia was 4, which means "Severe Akathisia" according to the BARS (Barnes Akathisia Rating Scale). The treatment of this symptom was complicated, but finally we observed a gradual improvement in the patient's akathisia after using a combination of drugs: clonazepam, propanolol, mirtazapine, biperiden and alpha-tocopherol. Suicidal thoughts and depressive symptoms disappeared after decreasing akathisia. This case report is an extremely rare and severe example of akathisia, considering the tardive onset of the presentation, its predominant upperbody involvement and its link with a suicide attempt. Akathisia causes major distress to the patient and may result in mood disorders and suicidal behavior. Further studies to understand the pathophysiology and treatment possibilities of akathisia are needed.

\section{REFERENCES:}

Azhar MZ, Varma SL. 1992. Akathisia-induced suicidal behaviour. Eur Psychiatry 7: 239-241.

Barnes T. 1989. Barnes Akathisia Rating Scale. Br J Psychiatry 54: 572-575.

Wright MT. Antiemetics, akathisia, and pregnancy. Psychosomatics. 2007; 48(6): 461-466. 